\title{
4 Interkulturelle Dialog-Räume für Beteiligung
}

○ Hier geht es zum Glossar.

m Hier geht es zu den Methoden, Artefakten und Werkzeugen.

$\hookrightarrow$ Hier wird das Thema weiter vertieft. 


\section{Kurzfassung}

Interkulturelle Dialog-Räume für Beteiligung in der Stadtentwicklung verstehen wir nicht als ,Zwischenräume' zwischen klar voneinander unterscheidbaren und unveränderlichen Kulturen. Vielmehr geht es um Räume, die für Menschen mit unterschiedlichen (Migrations-)Geschichten ohne Barrieren zugänglich sind. Zugleich sollten sie so gestaltet sein, dass sie die Beteiligten motivieren, miteinander in ODialog zu treten.

Solche Räume entstehen in der Regel nicht zufällig. Sie müssen proaktiv gestaltet werden. Ein offenes Beteiligungsangebot ,für alle' erreicht nur dann eine Vielfalt von Stadtnutzer*innen, wenn die unterschiedlichen Voraussetzungen und Barrieren für eine Teilnahme ausreichend reflektiert werden und sich das Ergebnis dieser Reflexion in der Gestaltung des Beteiligungsangebots niederschlägt. Unterschiedliche OMilieus und Kommunikationsstile bloß zu tolerieren oder wenigstens nicht aktiv auszugrenzen, reicht nicht aus. Es geht hier vielmehr um aktive Anerkennung. Insofern ist das Ziel eines interkulturellen Dialogs nicht ohne Einfühlungsvermögen, Engagement und Ressourcen (z. B. Personal und Zeit) zu erreichen.

Um beteiligungsferne Milieus zu aktivieren, können über Angebote ,für alle' hinaus aber auch zielgruppenspezifische Angebote sinnvoll sein. Solche Angebote geben die Gelegenheit, sich innerhalb einer Gruppe über gemeinsame Interessen und Bedürfnisse zu verständigen, ohne diese als, besonders' erklären zu müssen. Außerdem können sie Schutzräume für Gruppen bieten, die es nicht gewohnt sind, sich in der Öffentlichkeit zu äußern. Beteiligungsferne Milieus gibt es dabei nicht nur unter Menschen mit Migrationsgeschichte, sondern z. B. auch unter Jugendlichen und sozial benachteiligten Personen. Insofern beschränken sich interkulturelle Dialog-Räume nicht auf den Fokus Migration.

Die Fähigkeit zum interkulturellen Dialog ist eine Kompetenz, die Menschen im privaten Bereich ebenso wie bei ehrenamtlichem Engagement oder am Arbeitsplatz erwerben können. Als zur Gewohnheit gewordenes, verkörpertes'Wissen (Haraway, 
1988) kommt sie im privaten und beruflichen Alltag zum Einsatz. Unerfahrenheit mit interkulturellen Begegnungen kann einen Dialog blockieren. Ein Beispiel ist, dass Menschen sich oft aus Angst vor Fehlern auf vertraute Positionen zurückziehen, die durch Regelwerke und Routinen abgesichert sind, anstatt sich offen auf neue Perspektiven einzulassen.

Das Konzept der interkulturellen Integration geht davon aus, dass der gemeinsame soziale und kulturelle Rahmen des Zusammenlebens wandelbar ist und eine neue Normalität entstehen kann. Die dabei notwendigen Aushandlungsprozesse sind dynamisch und verlaufen nicht ohne Konflikte. Wenn interkulturelle Integration gelingen soll, braucht es Engagement und Anstrengungen auf allen Seiten, einen interkulturellen Dialog - auch in der Stadtentwicklung - selbstbewusst und aktiv zu gestalten. 


\section{Erkenntnisse aus der Fachdiskussion}

\section{Das Leitbild der interkulturellen Integration}

Zuwanderung ist kein neues Phänomen und in Deutschland inzwischen Normalität. Dennoch stellt sich die Frage nach der OTeilhabe verschiedener zugewanderter Gruppen immer wieder neu. Menschen, die erst kürzlich nach Deutschland zugewandert sind, unterscheiden sich sowohl untereinander als auch von solchen Gruppen, die bereits vor Jahrzehnten hierherkamen, oder von den nachfolgenden Generationen. Unterschiede beziehen sich z. B. auf ihre Präferenzen, Kompetenzen und Ressourcen bei der Nutzung und Aneignung städtischer Räume. Menschen mit Migrationsgeschichte sind nicht mehr und nicht weniger vielfältig als Menschen ohne Migrationsgeschichte (Schneider, 2020).

Das Konzept der ,interkulturellen Integration' aus der Migrationsforschung fordert von allen Mitgliedern einer Gesellschaft eine aktive Akzeptanz soziokultureller Unterschiede und gleichzeitig die Anerkennung eines gemeinsamen rechtlichen, kulturellen und sozialen Rahmens (Geißler \& Weber-Menges, 2013). Dieser Rahmen ist wandelbar und kann daher auch neu verhandelt werden. Eine aktive Akzeptanz bedeutet dabei nicht nur, Zuwanderung und Diversität einfach nur zu tolerieren. Sie meint vielmehr, diese als Potenzial zu begreifen und vorausschauend zu gestalten.

Dabei zeichnet sich ein, Integrationsparadox' ab (vgl. im Folgenden El-Mafaalani, 2018): Je erfolgreicher Integration voranschreitet, desto mehr Konflikte entstehen, weil nun Menschen ein gesellschaftliches Mitspracherecht erhalten, die vorher ausgeschlossen waren. Geht es zunächst vor allem darum, auch ,ein Stück vom Kuchen' (z. B. gleiche Bildungs- oder Karrierechancen) zu bekommen, wird später um den ,Kuchen' selbst gerungen, 
also um den gemeinsamen rechtlichen, sozialen und kulturellen Rahmen, der das Zusammenleben organisiert und regelt. In einer Gesellschaft, die von Zuwanderung und Vielfalt geprägt ist, sind Konflikte demnach ein gutes Zeichen für gelingende Integration - auch wenn das auf den ersten Blick widersprüchlich klingt. Integration bedeutet dann die Anerkennung, das Aushalten und das Verhandeln von Ambivalenzen und Konflikten (Foroutan, 2019, S. 24).

\section{Interkulturelle Dialog-Räume}

Das lateinische Wort ,inter' bedeutet ,zwischen‘. Das Wort Interkultur weckt deshalb die Erwartung, dass es um einen Zwischenraum zwischen feststehenden Kulturen geht - ähnlich wie früher $\bigcirc$,multikulti‘ für das Miteinander unterschiedlicher, meist ethnisch oder staatsbürgerlich definierter Gruppen stand.

Kultur meint hier jedoch ausdrücklich nicht, dass sich ethnische Gruppen entlang vermeintlich eindeutiger Merkmale voneinander unterscheiden lassen. Vielmehr meint der Begriff zum einen die unterschiedlichen Arten und Weisen, wie Menschen sich einen ,Reim' auf gesellschaftliche Verhältnisse machen (Hark \&Villa, 2017, S. 30) - geprägt u. a. von gelebten Traditionen, Erfahrungen, politischen und religiösen OInstitutionen sowie sozioökonomischen Verhältnissen. Zum anderen meint Kultur ein Prinzip der Organisation (Terkessidis, 2018, S. 10), z. B. im Sinne von Unternehmenskulturen.

Interkulturelle Dialog-Räume sind folglich keine Zwischenräume zwischen unterschiedlichen, klar abgrenzbaren Gruppen von Menschen. Es sind Räume, die für Menschen mit unterschiedlichen Perspektiven auf Gesellschaft barrierefrei zugänglich (Terkessidis, 2018, S. 130) und so gestaltet sind, dass ein Dialog zwischen ihnen aktiv befördert wird. Solche Räume werden umso bedeutsamer in ,postmigrantischen' Gesellschaften, d. h. Gesellschaften, die in ihren Strukturen und Alltagswelten von (andauernder) Zuwanderung geprägt sind (Hill \& Yildiz, 2018). 
Im INTERPART-Kontext sind interkulturelle Dialog-Räume in doppelter Hinsicht relevant: Beteiligung zu Themen der Stadtentwicklung kann zum einen selbst Kristallisationspunkt eines solchen Dialogs sein. Zum anderen sind Stadträume, also öffentliche und halb-öffentliche Orte im analogen und digitalen Raum (z. B. Plätze, Parks, Plattformen), potenzielle Orte interkulturellen Dialogs. Strategisch stellen sich zwei Fragen: Wie können solche Dialog-Räume initiiert und gestaltet werden? Und wie können unterschiedliche, z. T. höchst individuelle Barrieren erkannt und abgebaut werden? Solche Barrieren können materiell sein (wie Stufen zu einem Gebäude), aber auch räumliche und zeitliche Rahmenbedingungen, Sprache, Information und Orientierung (Terkessidis, 2018, S.151-155).

\section{Fokus auf Zielgruppen oder Normalisierung?}

Es gibt unterschiedliche Wege, um interkulturelle Dialog-Räume aktiv herzustellen. So können Stadtplaner*innen z. B. besondere Beteiligungsangebote für spezifische (benachteiligte) Bevölkerungsgruppen schaffen, um deren Mitsprache zu erweitern. Sie können aber auch Beteiligungsangebote ,für alle‘ so gestalten, dass diverse sprachliche, raumzeitliche, materielle oder sonstige Anforderungen von vornherein mitbedacht werden. Beide Wege haben Vor- und Nachteile (vgl. im Folgenden Boger, 2015; 2017).

Spezielle Angebote für benachteiligte oder stigmatisierte Gruppen können Solidarität und Kompetenzen der Beteiligten stärken und dazu befähigen, den eigenen Interessen in Stadtentwicklungsprozessen mehr Gehör zu verschaffen. In manchen gesellschaftlichen Milieus sind es z. B. Frauen nicht gewohnt, in der Öffentlichkeit zu sprechen. Entsprechende Trainings und Konsultationen in geschützten Räumen können ihnen mehr Sicherheit geben.

Für Oprivilegierte Gruppen wie z. B. akademisch geprägte Bürgerinitiativen können hingegen Reflexionsräume eröffnet 
werden, in denen sie sensibilisiert werden für Diskriminierungsprozesse und die eigenen Möglichkeiten, diesen entgegenzuwirken. Ein gruppenübergreifender Dialog findet damit allerdings zunächst nicht statt, und die ,normalen“ Beteiligungsangebote werden durch solche Formate nicht hinterfragt - obwohl sie mit Blick auf Interkultur auch anders gedacht werden könnten.

Beteiligungsprozesse in der Stadtentwicklung können jedoch auch so gestaltet werden, dass benachteiligte Gruppen gleiche Möglichkeiten, Rechte und Pflichten erhalten wie Teilnehmer*innen, die normalerweise eher privilegiert sind. Dass die Vielfalt der Beteiligten als normal betrachtet wird, kann sich z. B. darin äußern, dass auf unterschiedliche sprachliche, zeitliche, räumliche und materielle Bedürfnisse ganz selbstverständlich eingegangen wird. Fach- und Laienwissen sowie unterschiedliche Formen von Beteiligung und Engagement werden gleichermaßen anerkannt.

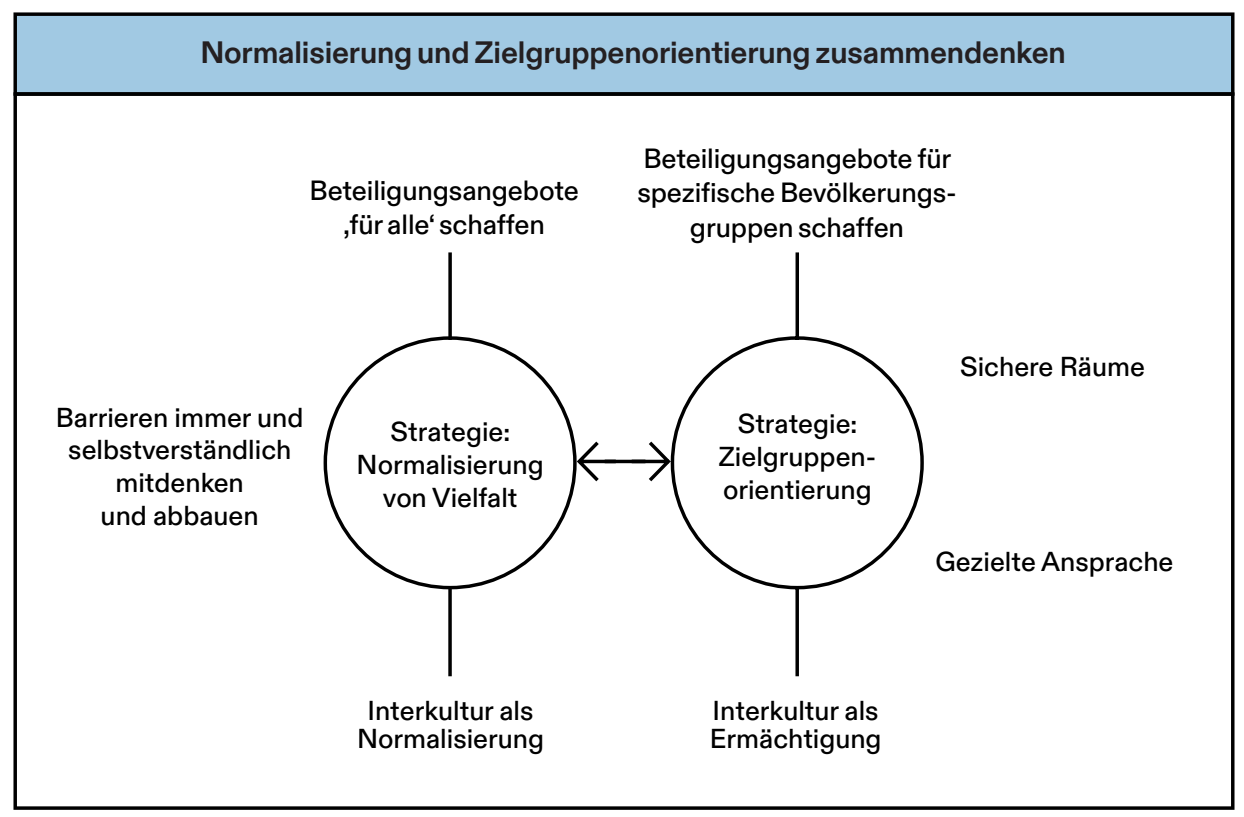

Der Versuch, beide Strategien - Betonung unterschiedlicher Zielgruppen und Normalisierung von Vielfalt - zu kombinieren, führt zwangsläufig zu Widersprüchen. Bei der Entscheidung, welcher 
Weg beschritten werden soll, ist zu berücksichtigen, welche Strategie die Beteiligten selbst befürworten. Denn jede Strategie kann für sich genommen dazu beitragen, interkulturellen Dialog kurz- oder mittelfristig zu befördern. Offene Fragen zu benennen, die sich durch die Wahl einer Strategie in einer Beteiligungssituation ergeben, ist eine wichtige Reflexionsaufgabe im interkulturellen Dialog.

\section{Denkraum}

Um interkulturelle Dialog-Räume der Partizipation in der Stadtentwicklung zu gestalten, ist es wichtig zu überlegen, welchem Zweck sie dienen sollen.

Notieren Sie selbst doch einmal in drei Stichpunkten, ob bzw. weshalb und wozu Sie einen interkulturellen Raum der Partizipation benötigen.

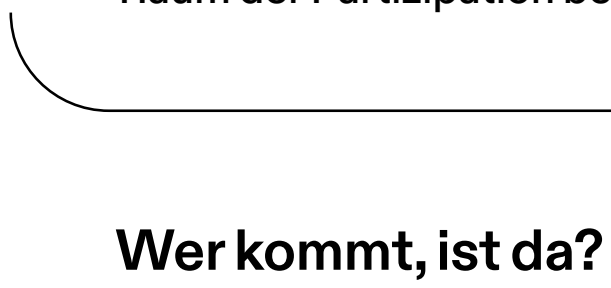

Viele Beteiligungsverfahren in der Stadtentwicklung sind als öffentliche Veranstaltungen geplant, zu denen alle Interessierten eingeladen werden. Ein Mitglied aus einer der beiden verwaltungsinternen Projektgruppen in INTERPART stellte in einem Interview fest:

„Das heißt nicht, dass es Veranstaltungen nur für Deutsche gibt, sondern dass es Veranstaltungen gibt und alle [einen] gleichberechtigten Zugang haben: Wer kommt, ist da." (\#hs_025)

Doch gerade solche Veranstaltungen sind häufig nicht für alle attraktiv, wenn sie bestimmte Kommunikationsstile nicht berück- 
sichtigen. Seit Jahren werden alternative, inklusivere Beteiligungsverfahren diskutiert und punktuell auch erprobt, z. B. im ,strategischen Integrationsmanagement" seit Anfang der 2000er Jahre (Gesemann, 2016, S. 284).

Vielfalt spiegelt sich häufig als politische Forderung in Zielen, Methoden und Instrumenten der Partizipation (Selle, 2019, S. 37). Zielgruppenspezifische Angebote werden bisher überwiegend im Programm ○, Soziale Stadt“ (seit 2020: „Sozialer Zusammenhalt") und in anderen Programmen der OStädtebauförderung umgesetzt. In anderen Bereichen der OStadtplanung sind sie kaum verbreitet. Einen ,interkulturellen Kodex‘ für Planungsprozesse gibt es bisher nur selten (Selle, 2019, S. 41).

Beteiligungsferne Milieus mit und ohne Migrationsgeschichte und das Wissen über ihre Alltagswelten sind daher in Beteiligungsangeboten der Stadtentwicklung häufig unterrepräsentiert (Friesecke, 2017). Diese stoßen z. B. dort an Grenzen, wo Menschen die (Amts-)Sprache Deutsch noch nicht gut sprechen oder am Verständnis von Fachsprachen scheitern.

\section{Interkulturelle Dialog-Räume in den INTERPART-Reallaboren}

\section{Erste Schritte}

Ziel von INTERPART war es, interkulturelle Dialog-Räume zu etablieren und sie für eine vielfältige Stadtgesellschaft barrierefrei zu gestalten, ohne dabei ausschließlich auf ethnische Merkmale zu schauen. Vielfältige Interventionen kamen in den Reallaboren auf experimentelle Weise zum Einsatz. Dabei spielten Gestaltungsprinzipien in mehrfacher Hinsicht eine große Rolle-von materiellen Räumen bis hin zu Installationen und OArtefakten. Über die Bedeutung materieller Gestaltung sprach bereits in 
einem unserer ersten DExpert*innen-Interviews ein OintermediärerTräger in Moabit:
„Also allein schon, wenn man in einen Raum kommt, und der Raum ist halt einfach nicht dafür gedacht, dass man sich da austauschen kann, dann ist das schon einmal eine Grundvoraussetzung, die einfach nicht gegeben ist. Das heißt, das ganze Drumherum muss halt auch stimmen." (\#cd_006)

Den Anspruch, einzelne Gruppen als Adressat*innen für Beteiligung zu gewinnen und zu stärken, setzte INTERPART vor allem durch das Erproben von Formaten um, die verschiedenen zivilgesellschaftlichen Gruppen mehr Gehör verschaffen sollten. Hierzu gehörten der DPodcast und das $\Phi$ Brettspiel. Darüber hinaus stellten die DErzählecke und die DErzählrunde sowie eine DKKlingel-Installation Ansätze dar, Vielfalt als Normalität und Selbstverständlichkeit der Kommunikation einzuplanen.

Ein weiteres experimentelles Format zur Eröffnung interkultureller Dialog-Räume war das Ko-Design eines OPrototyps für ein ФOnline-Beteiligungstool als zusätzlichem digitalen Beteiligungskanal. Er kann als Schnittstelle zwischen beteiligten Gruppen und Beteiligungsformaten dienen und in einem Beteiligungsprozess als, Gedächtnis‘ oder,Speicher' funktionieren.

Mit der Verwaltung diskutierten wir - über die konkreten Beteiligungsformate und Wege der Ansprache hinaus - Möglichkeiten einer interkulturellen Öffnung durch institutionellen Wandel, z. B. durch die abteilungsübergreifende Zusammenarbeit, eine entsprechende Ressourcenausstattung und Personalentwicklung ( $\hookrightarrow$ Kap. 8).

Dabei begleiteten uns die o. g. Ambivalenzen bei der strategischen Gestaltung interkultureller Dialog-Räume von Anfang an. In den מFachworkshops beider Städte und im WFachdialog zu Rassismus in den Institutionen kritisierten viele Beteiligte besondere Ansprache- und Beteiligungsformate für Menschen mit Migrationsgeschichte. Dies kommt in zwei Zitaten aus Wiesbaden und Berlin beispielhaft zum Ausdruck: 
„Es gibt bestimmte Traditionen und Kulturen in migrantischen Communities, die sich etwas abgrenzen, aber die Grundbedürfnisse sind mehr oder weniger die gleichen, weil es einfach genauso Menschen sind wie alle anderen. Und das war der [...] Grund, wieso ich mir gedacht habe: Nein, wieso Migranten extra? Was soll das denn für Migranten? Also jeder will gute Luft, Platz für seine Kinder zum Spielen und außerdem müssen sie das mit den Leuten vor Ort klären." (\#cd_001)

\begin{abstract}
„Also wir wissen zum Beispiel, dass das Milieu [der] Konsumhedonisten große Probleme hat, an Beteiligungsveranstaltungen teilzunehmen, und das ist unabhängig [...] von der ethnischen Herkunft. Also die migrantischen Milieus und die deutschstämmigen Milieus, sage ich jetzt mal, unterscheiden sich auch nicht groß und [...] haben die gleichen Probleme [...]. Auch die bürgerlichen Milieus, [...] unabhängig von migrantischer Herkunft oder nicht, haben [...] gute Zugänge zu Beteiligung. Also [es ist] eher von den Milieus abhängig als von der ethnischen Herkunft.“ (\#FD_RI)
\end{abstract}

Für andere Teilnehmer*innen hingegen war unstrittig, dass einzelne Gruppen mit Migrationsgeschichte durchaus ,anders' angesprochen werden müssen, z. B. aufgrund von fehlenden Erfahrungen mit dem deutschen Verwaltungssystem und unterschiedlichen Sprachen (\#FD_RI). 


\section{Selbstverständliche sprachliche Vielfalt: Die Klingel-Installation}

Die Kombination aus Klingel-Installation und Erzählecke ( $\hookrightarrow$ Kap. 6) bei der ersten מ Vor-Ort-Intervention stellte den Versuch dar, Alltagswissen und Vielfalt durch Mehrsprachigkeit sichtbar zu machen.

Die Klingel-Installation - zugleich ,Blickfang und crossmediale Benutzerschnittstelle-ermöglichte uns zu untersuchen, wie sich die persönliche Interaktion mit Menschen vor Ort mit Online-Formaten kombinieren lässt. Menschen, die unterschiedliche Sprachen sprechen, konnten sich hier zu einem gemeinsamen Thema in der Nachbarschaft äußern. Dabei war es offenbar nicht entscheidend, dass die Übersetzung zwischen den Sprachen nicht immer hundertprozentig stimmte. Schon das Angebot, verschiedene Sprachen als Einstieg in die Beteiligung auszuprobieren, wurde sehr gut angenommen. So wurde die Botschaft vermittelt, dass Vielfalt in der Beteiligung normal ist. Ein Ko-Forscher beobachtete folgendes Gespräch:
„Ein Mädchen sagt zu seiner Mutter: ,Mach auf Deutsch, Mama, mach auf Deutsch.' Beide bespre- chen dann auf Farsi, welche Sprache sie am Klingel- schild nutzen wollen. Die Mutter sagt schließlich: ,Das machen wir auf Deutsch.“”(\#rb_012).

Die Klingel-Installation veränderte den öffentlichen Raum materiell und machte das Aushandeln von sprachlicher Differenz in der Öffentlichkeit sichtbar, ohne sie zu bewerten. Durch die Alltäglichkeit von Hauseingängen und das damit verbundene Wissen der Stadtnutzer*innen, wie eine Klingel funktioniert, war die Hemmschwelle gering, die Klingel-Installation auszuprobieren. Als Mensch-Maschine-Schnittstelle rief sie auf verschiedenen Ebenenen - Mehrsprachigkeit, Technologie, Kommunikation Reaktionen hervor. Durch die computergestützte Übersetzung mithilfe künstlicher Intelligenz konnten so Sprachbarrieren über- 
wunden werden. Hier zeigt sich das Potenzial alltäglicher digitaler Kommunikationshilfen ( $\hookrightarrow$ Kap. 7). Der Austausch über die Übersetzungsfehler und über das Ausprobieren von Sprachen halfen bei der Aktivierung von Stadtnutzer*innen.

Allerdings entstand über die unmittelbare Klingel-Situation hinaus am Platz kaum ein thematisch fokussierter Dialog. Die Klingel-Installation wurde insgesamt vor allem spielerisch genutzt und diente auch zur Kontaktaufnahme mit Stadtnutzer*innen, die im Anschluss in der Erzählecke ihre individuellen Perspektiven auf die Nachbarschaft erläuterten. Konkret war sie ein inhaltlicher Anker, um Besucher*innen in die Vor-Ort-Intervention einzubeziehen und mit ihnen ins Gespräch zu kommen. Ein Mitglied der verwaltungsinternen Projektgruppe bezeichnete sie als „inspirierendes, spielerisches Format im Umgang mit Sprache“" (\#fs_001).

Aus Sicht des Projektteams war das Potenzial des Objekts für eine ,Kultur der Mehrsprachigkeit' damit noch nicht ausgeschöpft ( $\hookrightarrow$ Kap. 7). In einem nächsten Schritt wurde das Artefakt losgelöst vom Motiv des Tors weiterentwickelt und eine transportablere Version der Klingel entworfen, die als Objekt anpassbar und individuell bedienbar war. Geplant war, die KlingelInstallation in weiteren Ko-Forschungs-Workshops zu nutzen, um eine kritische Diskussion über die Anforderungen an interkulturellen Dialog anzuregen. Dabei sollten die Erfahrungen aus den Vor-Ort-Interventionen aufgegriffen werden. Hier hatten sich mehrsprachige Angebote auf unterschiedlichen Ebenen-Texte, Installation, Workshop und Sprachmittler*innen - als hilfreicher Beitrag zu einer wertschätzenden Kommunikation im interkulturellen Dialog erwiesen. Aufgrund der Pandemie konnten die Workshops jedoch nicht mehr wie geplant durchgeführt werden, sodass stattdessen das digitale Format der Podcasts zum Einsatz kam (s. u. und $\hookrightarrow$ Kap. 6).

Ein kritischer Aspekt bleiben die in künstliche Intelligenz und andere Technologien eingeschriebenen Machtverhältnisse, die durch die Anwendung reproduziert werden. Ein pragmatischer Umgang mit verfügbaren Werkzeugen ist oft schon aus finanziellen Aspekten und Gründen der einfachen Handhabung geboten. Gleichzeitig sind die Grenzen und Einschränkungen, die mit dem 
Einsatz spezieller Technologien - von bestimmten Herstellernverbunden sind, in ihrer Bedeutung für den Dialog immer wieder zu hinterfragen.

\section{"Beteiligung, wie ich sie mir wünsche": Workshop mit Brettspiel}

Für die zweite Vor-Ort-Intervention in Biebrich entwarfen wir ein Brettspiel, das die Teilnehmer*innen im Rahmen eines Workshops für Beteiligung aktivieren sollte. Zudem wollten wir darüber ihre Vorstellungen von einer optimalen Beteiligungssituation kennenlernen. Durch das Brettspiel wurde vorhandenes Wissen aktiviert, welches im Gespräch wohl eher verborgen geblieben wäre. Alle Arbeitsschritte waren in türkischer, arabischer und deutscher Sprache auf Plakaten an Stellwänden erklärt, und Sprachmittler*innen standen für Erläuterungen aufTürkisch, Arabisch und Kurdisch zur Verfügung.

Geplant war, mit dem Brettspiel den Anlass für einen Dialog zwischen Menschen zu schaffen, die sich vorher nicht kannten. Letztlich waren es jedoch vor allem zwei existierende, bereits miteinander vertraute Gruppen, die das Brettspiel erprobten. Workshop 1 fand in türkischer Sprache mit einer Frauengruppe statt und wurde für die deutschsprachige INTERPART-Forscherin übersetzt. Dagegen waren fast alle Teilnehmer*innen in Workshop 2 Mitglieder einer lokalen Bürgerinitiative und hatten beruflich mit Architektur und Stadtplanung zu tun.

Gruppe 1 erschien bei der Veranstaltung mit dem festen Ziel, ihre Anliegen zu formulieren - und mit der Erwartung, gehört zu werden. Das Angebot einer niedrigschwelligen, spielerischen Interaktion schien fast deplatziert angesichts der Klarheit und Konkretheit der Anliegen: mehr Nachhilfeunterricht-Angebote für ihre Kinder und besserer Zugang zu bezahlbaren Mietwohnungen. Die Vorstellung der Gruppe war klar darauf gerichtet, dass eine Olnstitution sich ihrer Anliegen annimmt. Ehrenamtliche Vernetzung und Selbstorganisation wurden kaum diskutiert. 


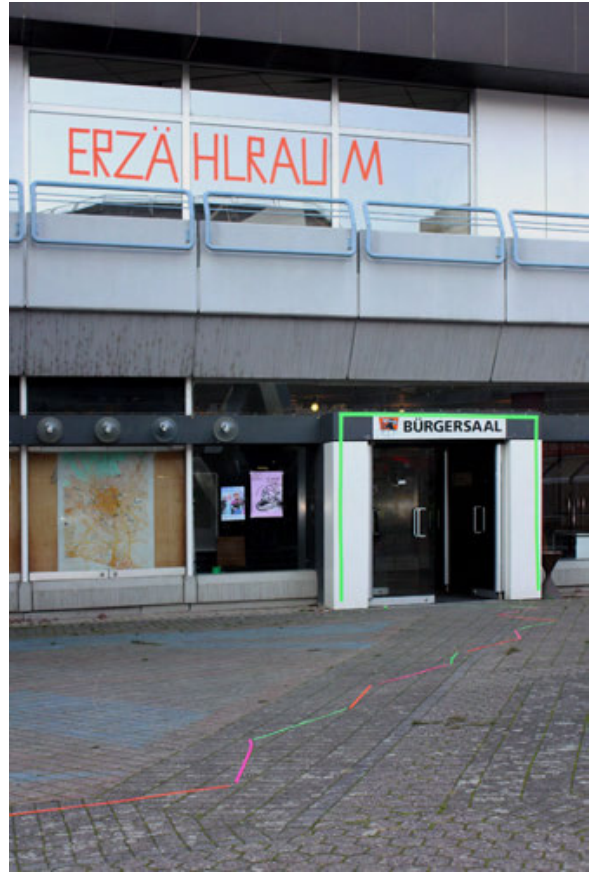

Vor-Ort-Intervention im Bürgersaal, Wiesbaden-Biebrich

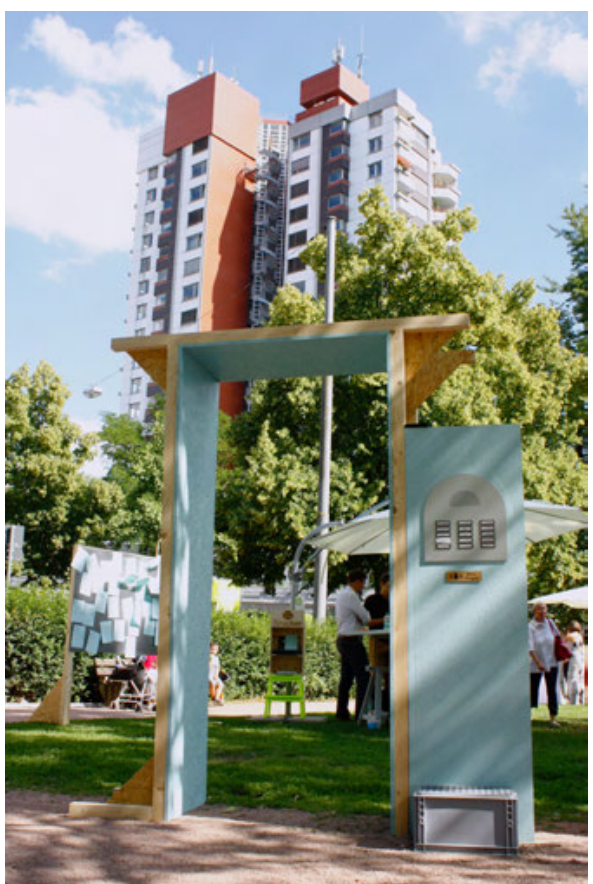

Vor-Ort-Intervention vor der Galatea-Anlage, Wiesbaden-Biebrich 
Gruppe 2 hingegen ließ sich auf die spielerische Situation mit Neugierde, aber auch einem hohen $\mathrm{Maß}$ an Abgeklärtheit ein. Vermutlich verfügten die Mitglieder bereits über berufliche Erfahrungen mit solchen Workshops und Planspielen. Fragen der strategischen Vernetzung wurden mit großem Selbstbewusstsein diskutiert und geeignete lokale Institutionen als Ansprechpartner*innen ganz selbstverständlich benannt. Die Gruppe entwickelte eine elaborierte Strategie zum Einsatz von Medien und Präsenz-Formaten zur Ansprache unterschiedlicher Zielgruppen. Sie formulierte außerdem ihren Wunsch, Mitgestaltung durch ein strukturiertes Vorgehen zu befördern. Dabei zeigte sich deutlich das Interesse der Gruppe, sich als Akteur der Stadtentwicklung zu etablieren.

Die COVID-19-Pandemie verhinderte die Durchführung weiterer geplanter Workshops mit dem Brettspiel. Doch schon diese Erfahrungen zeigen die große Spannbreite zwischen Wünschen an Beteiligung auf. Gruppe 1 brachte praktische, alltagsnahe Anliegen vor, traute sich selbst wenig bei deren Bearbeitung zu und suchte nach zuständigen Ansprechpartner*innen. Die Beteiligungsperspektive der zweiten Gruppe hingegen bezog sich darauf, selbst regelmäßig und themenübergreifend über die Entwicklung des Stadtteils mitzubestimmen. Dies zeigt deutlich, dass die Voraussetzungen für interkulturellen Dialog in Beteiligungsangeboten ,für alle‘ erst geschaffen werden müssen. Wir versuchten daher - wie der folgende Abschnitt zeigt - eine aktive Vernetzung lokaler Akteure im Stadtteil zu erreichen. 


\section{Interkulturelle Vernetzungsarbeit für lokale Partnerschaften}

Um Menschen für die INTERPART-Aktivitäten zu gewinnen, luden wir nicht nur öffentlich zu den o. g. Aktivitäten ein. Vielmehr baten wir wichtige Akteure in den Stadtteilen persönlich um Mitwirkung und das Weiterleiten unserer Einladung an weitere Initiativen. INTERPART-Forscher*innen suchten das Gespräch mit lokalen Gruppen, deren Selbstbeschreibung sich im weitesten Sinne auf Stadt(teil)entwicklung oder Zuwanderung bezog. Sie gingen außerdem an Orte, an denen sich die Vielfalt der Stadtgesellschaft zeigt, z. B. Schulen, Stadtteilzentren, Bildungs- und Sportvereine, religiöse Orte, politische und kulturelle Vereine und Institutionen, aber auch geschützte Räume wie Frauen-Cafés. Ziel war es, Ansätze, Orte und Akteure für interkulturellen Dialog in den Stadtteilen zu finden und zu vernetzen.

Im Rahmen von INTERPART aktivierte eine Ko-Forscherin der Wiesbadener Stabsstelle Identität. Engagement. Bürgerbeteiligung. Bevölkerungsgruppen und zivilgesellschaftliche Initiativen mit Migrationsgeschichte für Beteiligungsveranstaltungen der lokalen Stadtentwicklungsgesellschaft (SEG), um die Umsetzung des Programms „Soziale Stadt“ im Stadtteil Biebrich zu unterstützen. Das später eingesetzte $\bigcirc$ Quartiersmanagement sollte von dieser Netzwerkarbeit profitieren. In den Fachworkshops wurde immer wieder thematisiert, dass es im Verwaltungsalltag kaum möglich sei, eine solche Vernetzung dauerhaft zu leisten ( $\rightarrow$ Kap.8).

Das Programm „Berlin entwickelt neue Nachbarschaften“ (BENN) hatte hingegen das Ziel, Bewohner*innen und Geflüchtete in Moabit miteinander zu vernetzen. Das Team vor Ort nutzte bereits soziale Medien und Fotografie für Storytelling und zur Aktivierung für interkulturelle Begegnungen. In den Vor-OrtInterventionen entwickelten wir gemeinsam den Rahmen für einen interkulturellen Dialog über Stadtentwicklung, insbesondere durch die Erzählformate. 
KAPITEL 4: INTERKULTURELLE DIALOG-RÄUME

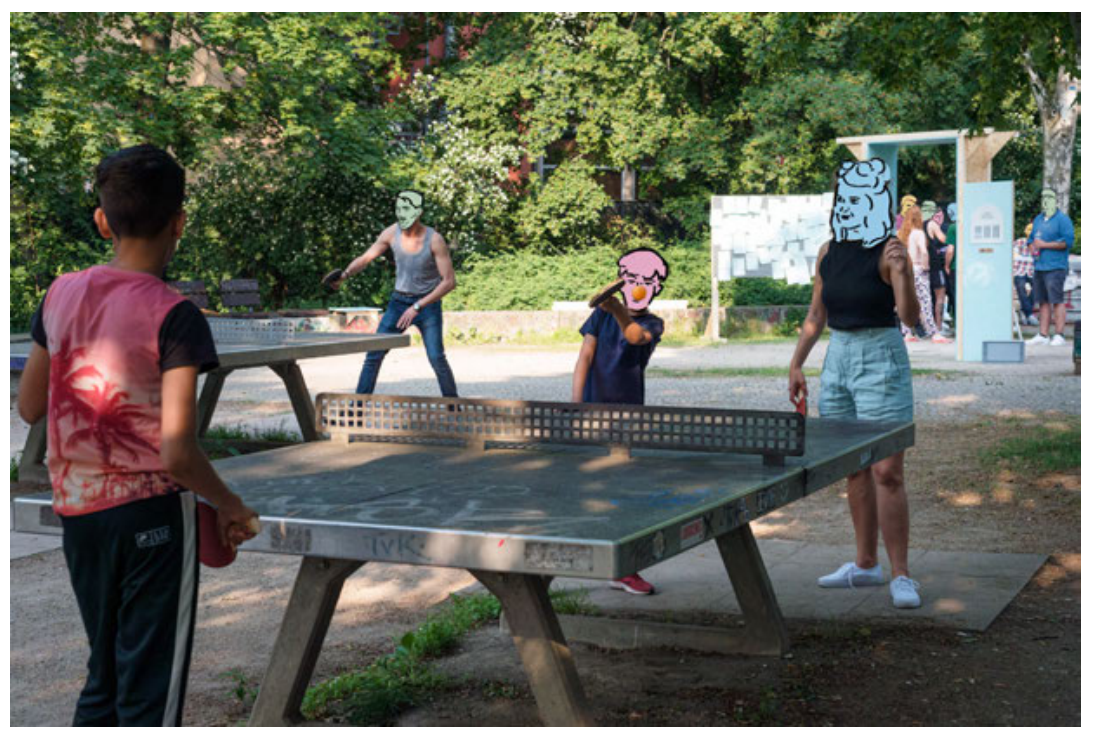

Tischtennisturnier als Teil der Vor-Ort-Intervention, Berlin-Moabit

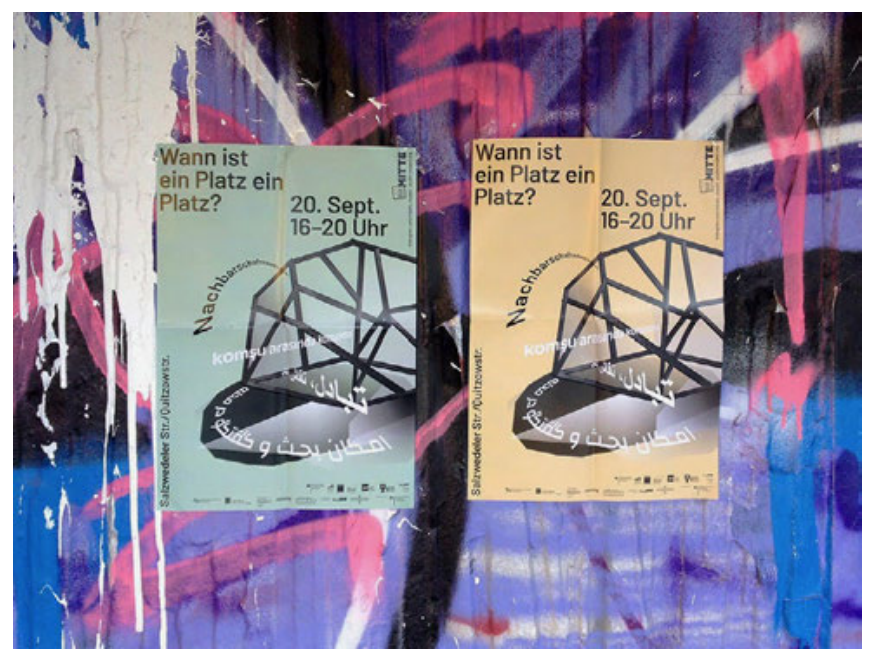

Mehrsprachiges Plakat zur Ankündigung der Vor-Ort-Intervention, Berlin-Moabit 
Auch wenn sich die Vernetzungsansätze deutlich voneinander unterschieden, nutzten wir schließlich ein gemeinsames Podcast-Format, um in beiden Stadtteilen interkulturellen Dialog zu unterstützen. Wir wollten mit Expert*innen, die bereits praktisch mit den Fragen zur interkulturellen Beteiligung zu tun haben, ins Gespräch kommen. Über das Medium Podcast wollten wir auch solches Wissen aktivieren, das normalerweise nicht explizit wird. Pandemiebedingt konnten keine Gruppen-, sondern nur ZweierGespräche stattfinden ( $\rightarrow$ Kap. 6).

Ziel war es, dass die Teilnehmer*innen den fertig produzierten Podcast als öffentlich hörbares Ergebnis auch mitnehmen können. Damit war der Podcast Ausdruck der Ko-Forschung. Migrantische Stimmen sollten bewusst gestärkt werden. Einige Teilnehmer*innen nutzten den Podcast im Nachgang, um ihre Arbeit online zu bewerben. In einem Auswertungsgespräch zeigte sich, dass Selbstreflexion und ein Nachdenken über unterschiedliche Positionen in der Stadtentwicklung durch den Podcast-Dialog angeregt wurden.

\section{Reflexion}

Die Frage, ob der Ansatz einer ,Beteiligung für alle‘ automatisch zu interkulturellen Räumen der Partizipation führt, wurde auch in den Reallaboren von INTERPART kontrovers diskutiert. Ist es dafür ausreichend, sich auf Themen und Nutzungsinteressen zu konzentrieren und Ansprache und Angebot methodisch inklusiv zu gestalten?

Die Leitlinien für Beteiligung in Berlin („,Gemeinsam Stadt machen!“, SenSW, 2019) und die Leitlinien in Wiesbaden („Bürger. Macht.Mit", Landeshauptstadt Wiesbaden, 2015), die im Sommer 2021 überarbeitet werden sollen, zielen auf eine größere Methodenvielfalt und punktuell mehrsprachige Informationen. Darüber hinaus sollen bis dato unterrepräsentierte Gruppen zukünftig in den Beteiligungs- und Begleitgremien repräsentiert sein. Über Sozialraum-, Milieu- und lokale Netzwerkanalysen sollen die 
Beteiligungsangebote stärker auf die lokale Bevölkerung zugeschnitten werden. Die Leitlinien und die dazugehörigen Aktivitäten spielen für die Etablierung interkulturellen Dialogs eine wichtige Rolle. Vor allem zeigen sie die Selbstverpflichtung von Politik und Verwaltung, Beteiligung auszubauen.

Es besteht allerdings die Gefahr, dass diese Ansätze zu stark vorstrukturiert sind, um tatsächlich Dialoge zu initiieren. Niedrigschwelligkeit bedeutet, sich auf unterschiedliche Zugänge zu Stadtentwicklung einzulassen und den Dialog tatsächlich zu wollen. Schwierig wird es, wenn Angebote sich an migrantische Stadtnutzer*innen als vermeintlich homogene Gruppe wenden und dieser Gruppe Fähigkeiten oder Interesse an Beteiligung pauschal zu- oder absprechen. Kritik an einer solchen defizitorientierten Position gab es auch unter unseren Ko-Forscher*innen, wie der folgende Auszug aus einem Protokoll des Fachdialogs zu Rassismus in den Institutionen zeigt:
„Das ist einmal das Bild, das wir transportieren von Migranten, [...] das ist meistens sehr defizitär. Das Bild in Verwaltung von Migranten-Eltern, die ihre Kin- der nicht zum Schwimmunterricht schicken und so-das hat immer etwas mit Problemen zu tun. Und auch in unseren Bildern oder Veröffentlichungen: Welche Menschen, welche Menschen mit Migrati- onsgeschichte stellen wir dort dar?"(\#FD_RI)

Interkultureller Dialog in der Beteiligung erfordert demnach einerseits, dass Zielgruppen nicht nach Herkunft, Geschlecht oder Alter charakterisiert und getrennt voneinander angesprochen werden. Andererseits muss, Augenhöhe' bewusst und in jeder Phase des Prozesses aktiv hergestellt werden. Nur so können vermeintlich ,besonders' erscheinende Barrieren selbstverständlich mitgedacht und langfristig abgebaut werden.

Gleichzeitig legen die bisherigen Erfahrungen in der Beteiligungspraxis nahe, dass es weiterhin auch Bedarf an Veranstaltungen und Formaten geben wird, die Bevölkerungsgruppen getrennt voneinander ansprechen. Um das eine vom anderen 
unterscheiden zu können, ist es unerlässlich, Nähe und Kontakt zu den jeweiligen Bevölkerungsgruppen möglichst kontinuierlich sicherzustellen und Bedarfe bzw. Kapazitäten zu eruieren. Der Hinweis aus den Verwaltungen, dass dafür schlicht die Ressourcen fehlen, wirft die Frage auf, wie eine strategische Neuorientierung hin zu stärkerer interkultureller Öffnung der administrativen Ebenen befördert werden kann ( $\hookrightarrow$ Kap. 8). 